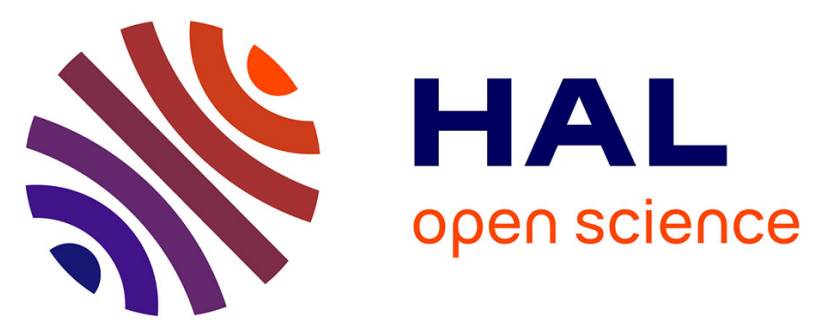

\title{
Three-Dimensional Investigation of Free-Edge Effects in Laminate Composites Using X-ray Tomography and Digital Volume Correlation
}

Pauline Lecomte-Grosbras, Julien Réthoré, Nathalie Limodin, Jean-Francois Witz, Mathias Brieu

\section{To cite this version:}

Pauline Lecomte-Grosbras, Julien Réthoré, Nathalie Limodin, Jean-Francois Witz, Mathias Brieu. Three-Dimensional Investigation of Free-Edge Effects in Laminate Composites Using X-ray Tomography and Digital Volume Correlation. Experimental Mechanics, 2015, 55 (1), pp.301-311. 10.1007/s11340-014-9891-1 . hal-01205922

\section{HAL Id: hal-01205922 \\ https://hal.science/hal-01205922}

Submitted on 10 May 2021

HAL is a multi-disciplinary open access archive for the deposit and dissemination of scientific research documents, whether they are published or not. The documents may come from teaching and research institutions in France or abroad, or from public or private research centers.
L'archive ouverte pluridisciplinaire HAL, est destinée au dépôt et à la diffusion de documents scientifiques de niveau recherche, publiés ou non, émanant des établissements d'enseignement et de recherche français ou étrangers, des laboratoires publics ou privés. 


\title{
Three-Dimensional Investigation of Free-Edge Effects in Laminate Composites Using X-ray Tomography and Digital Volume Correlation
}

\author{
P. Lecomte-Grosbras · J. Réthoré • N. Limodin · \\ J.-F. Witz • M. Brieu
}

\begin{abstract}
Laminate composite materials are widely used for many industrial applications because of their high specific mechanical properties as well as the possibility to adapt the structure properties to its application and loading conditions by optimization of the stacking sequence. It is now well known that the mechanical properties mismatch of adjacent layers having different fibre orientation induces stresses concentrations at interlaminar interfaces in the vicinity of structure edges. Edge effects experimental investigation is generally made through two dimensional measurements, crack initiation detection and characterization using various observation techniques. In this paper, it is proposed to investigate edge effects by coupling $2 \mathrm{D}$ and 3D measurements, using X-Ray tomography and kinematic field measurements. A preconditioned Digital Volume Correlation is developped in order to capture the in depth evolution of the residual displacement discontinuity at the ply interfaces. After it has been validated by comparison with the 2D measurements on the surface, the analysis of the $3 \mathrm{D}$ fields is carried out.
\end{abstract}

Keywords Laminate composite - Edge effects . $\mathrm{X}$-ray tomography $\cdot$ Digital volume correlation . Digital image correlation

P. Lecomte-Grosbras · N. Limodin · J.-F. Witz · M. Brieu Laboratoire de Mécanique de Lille, Ecole Centrale de Lille, CNRS UMR 8107, Cité scientifique, 59651

Villeneuve d'Ascq, France

P. Lecomte-Grosbras

e-mail: pauline.lecomte@ec-lille.fr

J. Réthoré $(\bowtie)$

LaMCoS, Université de Lyon, INSA Lyon, CNRS UMR 5259,

27 Avenue Jean Capelle, 69621 Villeurbanne, France

e-mail: julien.rethore@insa-lyon.fr

\section{Introduction}

Laminate composite materials are nowadays widely used for many industrial applications because of their high specific properties as well as the possibility to adapt the structure properties to its application and loading conditions by optimization of the stacking sequence. Because of the complexity of composite structures which are intrinsically highly heterogeneous, the prediction of the failure appearance remains a difficult question for the design of composite structures. It is now well known, see [1], that the mechanical properties mismatch of adjacent layers having different fibre orientation induces stress concentration at interlaminar interfaces in the vicinity of structure edges. This phenomenon induces a complex tridimensional stress state which cannot be predicted by the Classical Laminated Theory (CLT), as generally used for the design of composite structures. This may induce early delamination for critical stacking sequences as reported in [2]. Many studies have investigated the free edge effects in composite structures. Analytical and numerical works have been proposed to predict such a phenomenon [3-5]. However, experimental data are required to validate such approaches.

\section{Mechanical Analysis of Free-Edge Effects}

Edge effects experimental investigation is generally made through two dimensional measurements, crack initiation detection and characterization using various observation techniques. Indeed, previous experimental studies were performed using full field measurements, Moiré interferometry [6-8] or Digital Image Correlation (DIC) [9, 10], on the composite edges. The initiation of delamination is usually studied using acoustic techniques to detect the crack 
initiation $[11,12]$. The induced cracks are observed using optical or Scanning Electron Microscopy techniques [13]. $\mathrm{X}$-Ray radiographic observations have also been performed to assess the delamination surfaces or matrix cracks appearance inside damaged composite structures in [14]. However, to be completely highlighted, such a complex phenomenon needs 3D data in the bulk which are not easy to assess. Edge effects lead to 3D mechanical states and thus need 3D experimental characterization. With the recent development of high resolution X-Ray computed tomography it is now possible to reconstruct the 3D microstructure of composites as well as 3D cracks observations [15]. Moreover, the advances in image and volume analysis make it possible, on suitable textures, to measure the three dimensional displacement field (inside the volume) by Digital Volume Correlation (DVC) [16].

In this study, it is proposed to investigate edge effects by coupling 2D and 3D measurements, using X-Ray tomography and kinematic field measurements. The edge effect phenomenon will be detailed in a first section. The studied composite material and the experimental procedure, consisting in measuring 2D displacement fields during tensile loading and coupling with 3D observations and 3D residual displacement measurements using X-Ray tomography, will then be described. Afterwards, the image and volume correlation mathematical formulation will be detailed. Finally, results will be exposed and discussed.

Edge effects are highly localized stresses appearing in laminated composites at interlaminar interfaces where there is material properties discontinuity between layers [1]. In this study, we consider the case of a plane laminated structure made of unidirectional plies, assuming an homogeneous transverse isotropic elastic linear behaviour. The study of such a structure requires to introduce two reference frames: the first one for the structure $(x, y, z)$, and the second one for the material $(1,2,3)$. The direction 1 represents the fibres direction that makes an angle $\theta$ with the $z$ direction, and the axes 3 and $x$ are combined as shown in Fig. 1.

The differences of fibre orientation between adjacent plies are inducing mismatches of mechanical properties in the structure reference frame which lead to high interlaminar stress concentrations. For instance, a uniaxial tension in the $z$ direction applied on a laminate with a $\left[\theta_{n} /-\theta_{n}\right]_{s}$ stacking sequence, induces the stresses $\sigma_{z z}$ and $\sigma_{y z}$. The first one is due to the applied loading like in homogeneous materials and the second one is induced by the $\theta$ angle of the fibre in each ply. However the free edge condition

$\sigma . n=0$

applied on the $(x, z)$ plane of normal $n=y$ leads to a vanishing $\sigma_{y z}$ at the free edge. This free edge condition induces a variation of $\sigma_{y z}$ along the $y$ direction which gives rise to $\sigma_{x z}$ interlaminar stress concentration to satisfy the balance of momentum equations

$\left\{\begin{array}{l}\sigma_{x x, x}+\sigma_{x y, y}+0=0 \\ \sigma_{y x, x}+\sigma_{y y, y}+0=0 \\ \sigma_{x z, x}+\sigma_{y z, y}+0=0\end{array}\right.$.

In the present case $\sigma_{x x}=\sigma_{x y}=\sigma_{y y}=0$ because of the uniaxial loading and $\sigma_{i j, z}=0$ due to Saint-Venant's conditions. The balance equations are simplified as follows:

$\left\{\begin{aligned} 0+0+0 & =0 \\ 0+0+0 & =0 \\ \sigma_{x z, x}+\sigma_{y z, y}+0 & =0\end{aligned}\right.$

The combination of free edge and equilibrium conditions leads to complex tridimensional stress states with high stress concentrations near interlaminar interfaces on free edges which may induce early delamination of the laminated structure.

\section{Material and Experimental Procedure}

\section{Material}

The studied material is a Glass Fibre Reinforced Polymer (GFRP) composite made of unidirectional E-glass fibres and M9 epoxy matrix manufactured by Hexcel Company. The fibres have a diameter of $20 \mu \mathrm{m}$ and the ply thickness after polymerisation is $975 \mu \mathrm{m}$. Laminate plates of
Fig. 1 Samples geometry (in $\mathrm{mm}$ ) and reference frames
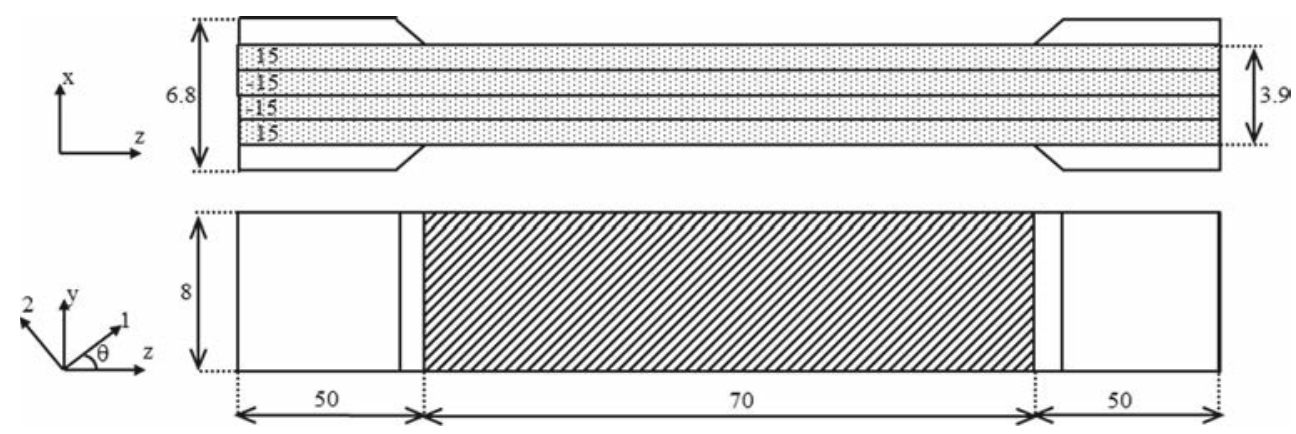
$\left[15^{\circ} /-15^{\circ}\right]_{s}$ stacking sequence have been manufactured. The geometry of the samples is shown on Fig. 1. This stacking sequence has been chosen because high shear stress $\sigma_{x z}$ concentrations appear at $-15^{\circ} / 15^{\circ}$ interlaminar interfaces due to edge effects [9]. The macroscopic behaviour of the laminate is linear up to failure with an equivalent modulus of $42 \mathrm{GPa}$ as shown on Fig. 2. Even if the macroscopic behaviour is linear, non-linear phenomena localized at interlaminar interfaces can be early induced by the edge effects and they can initiate the delamination of the laminated structure.

\section{Experimental Protocol}

To highlight free edge effects and localized delamination, it is necessary to perform the observations and analysis at the appropriate scale. The mechanical state being tridimensional it is also necessary to perform tridimensional experimental characterization in the volume. Samples have been tested in uniaxial tension, as presented in Fig. 3. As explained in Fig. 4, loading and unloading cycles of growing amplitude are combined with 2D observations with an optical camera and 3D observations using X-Ray tomography.

$\mathrm{X}$-Ray tomography acquisitions were performed using a Computed X-Ray Tomography (CT) system Phœnix VTomex (GE) fitted with a nanofocus tube whose acceleration voltage can be adjusted up to $160 \mathrm{kV}$. This technique consists in applying an incident X-ray beam to the sample and observing the transmitted beam converted into visible light and recorded by a detector. This recorded beam corresponds to a projection of the sample which is rotated over $360^{\circ}$ to record a sufficient number of projections to reconstruct the whole volume [17]. The contrast obtained in the reconstructed volume, which depends on the attenuation coefficient of the different microstructural elements,

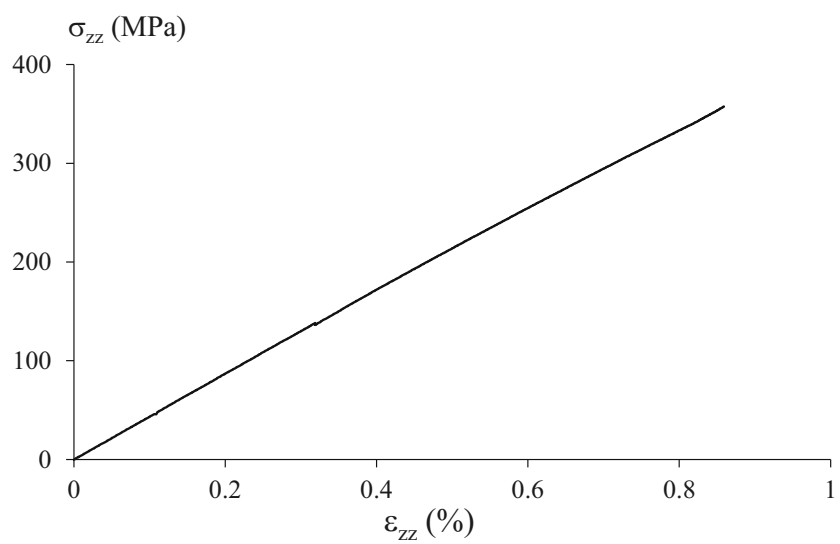

Fig. 2 Macroscopic stress-strain curve in uniaxial tensile loading of $\left[15^{\circ} /-15^{\circ}\right]_{s}$ laminated GFRP composite

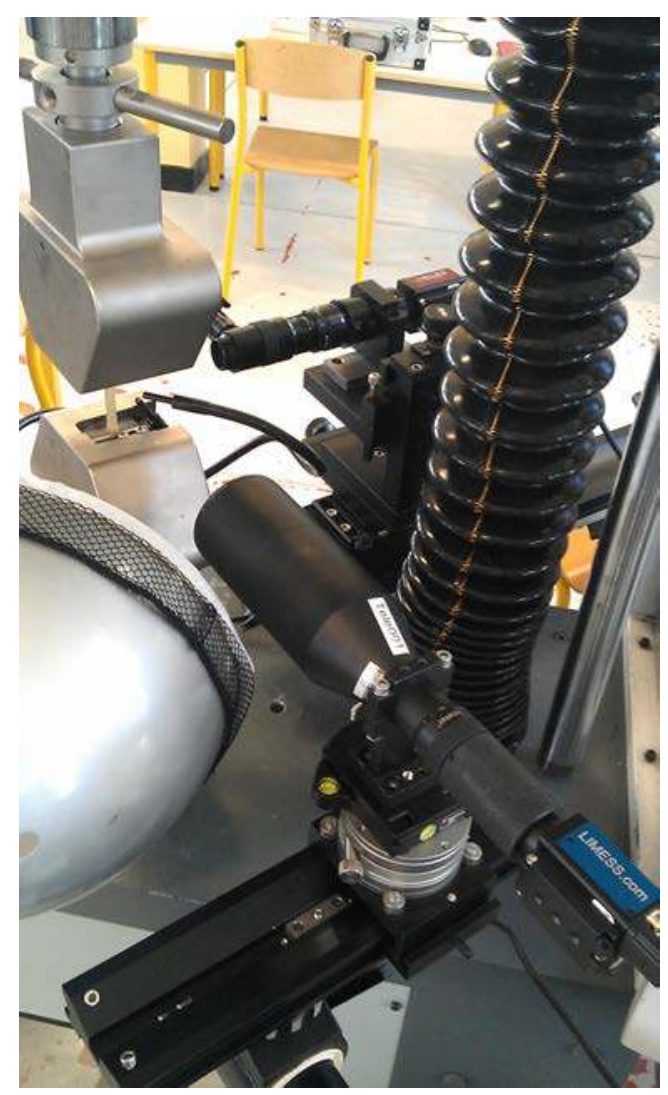

Fig. 3 Experimental setup

is high enough in GFRP composites for performing DVC analysis. In the present study, a $60 \mathrm{kV}$ acceleration voltage was selected to ensure a $10 \%$ transmission of the X-ray beam through the cross-section $(8 \times 3.9 \mathrm{~mm}$, see Fig. 1$)$ of the sample. The specimen was placed on a rotating stage in the tomography chamber between the X-ray source and an amorphous silicium diode array detector of dimensions $1920 \times 1536$ pixels. The smallest voxel size that could be achieved considering the specimen dimensions and the detector size is $4.5 \mu \mathrm{m}$. A set of 1500 projections (a scan) were taken while the sample was rotating over $360^{\circ}$ along its vertical axis. With an acquisition time per image of $500 \mathrm{~ms}$, one scan lasts about $50 \mathrm{~min}$. Reconstruction of the tomographic data was performed with a filtered back-projection algorithm using Phœnix datos|x 2.0 software developed by GE. Some artefacts that occur in lab CT, namely beam hardening due to the polychromatic nature of the beam, ring artefact due to non linear response of the detectors pixels, drift and artificial image dilation due to focal spot instability and tube dilation were reduced using the available software tools: automatic beam hardening correction, ring reduction using detector random shift during the acquisition and automatic compensation for drift and dilation effects that occur during a long scan. To have a good texture to perform DIC measurements, black and white speckle pattern is applied on 
Fig. 4 Experimental protocol:initial volume $V_{0}$, volume at step $n V_{n}$, initial image $I_{0}$, image at loading step $n I_{n}^{l}$ and image at unloading step $n I_{n}^{u}$

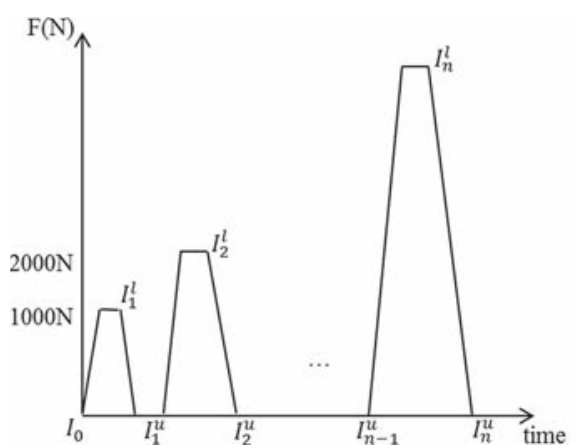

the observed edges. However, in the volume it is not possible to create an artificial texture inside the sample to have a suitable gray level distribution. In GFRP composite, the contrast between the glass fibres and the epoxy matrix is sufficient to observe the microstructure and to allow performing DVC measurements. Figure 5 shows the 2D images at macroscopic $((y, z)$ plane $)$ and mesoscopic scales $((x, z)$ plane) as well as the texture obtained in the volume by $3 \mathrm{D}$ $\mathrm{X}$-Ray tomography in the 3 orthogonal planes.

Once an initial observation of the volume $V_{0}$ is performed, the sample is fixed on the tensile machine and optical images are taken with a CCD camera attached to the tensile machine (see Fig. 3): first, at the free stress - free strain state, $I_{0}$, and then, at each loading, $I_{n}^{l}$, and unloading step, $I_{n}^{u}$. The images are acquired at the macroscopic scale in the $(y, z)$ plane (pixel size $11 \mu \mathrm{m}$ ), ie along the specimen length, and at the mesoscopic scale in the $(x, z)$ plane (pixel size $1.5 \mu \mathrm{m}$ ), perpendicular to the plies direction. The displacement field is measured for each image $I_{n}$ by DIC with the initial one, $I_{0}$, as a reference. When residual displacements are measured at an unloading step, between $I_{0}$ and $I_{n}^{u}$, the sample is removed from the tensile machine and

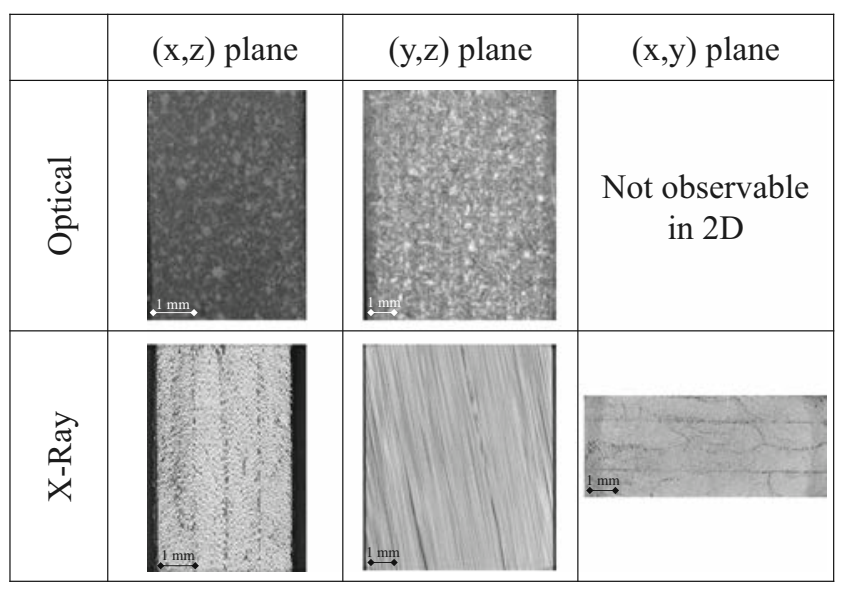

Fig. 5 Texture of 2D optical and 3D X-Ray images in various observation planes the damaged volume, $V_{n}$, is scanned by X-Ray tomography. Then the 3D displacement field is computed in the whole volume of the sample using DVC with the initial volume $V_{0}$ acquired in the undeformed state as a reference (see Fig. 4). It has been demonstrated in [18] that the use of a lab CT with a conical X-ray beam induces artefacts on the displacement field measured by DVC. Especially, the thermal dilatation of the X-ray source tube unit induces a variation of the distance between the source and the sensor resulting in an artificial affine transformation when measuring the displacement between two scans. In the proposed experimental protocol, several hours may pass between two successive scans, the state of the X-ray tube may thus be significantly changed. To reduce this effect, a warm-up of the tomograph is set up before each scan of the specimen. It consists in acquiring a series of unused radiographs during 15 minutes before starting the effective scanning of the specimen. From the results in [18], it may be considered that a stabilized thermomechanical state of the X-ray tube is obtained before each scan, thus limiting the underlined artificial affine tranformation. Although lessened, the instability of the X-ray source still needs to be compensated during long acquisition time. The datos $\mid \times 2.0$ software allows an initial scan with few projections to be recorded with a scan duration so short that the $\mathrm{X}$-ray source could be considered stable. This preliminary scan is then used at the reconstruction step to correct the projections of the actual scan.

\section{Digital image and Volume Correlation}

From the images (2D at loading and unloading stages and $3 \mathrm{D}$ at unloading stages) acquired during the experiments, displacement field measurements are performed by digital image or volume correlation. The method used herein is based on a finite element description of the displacement field as proposed initially by [19] in 2D and then extended to $3 \mathrm{D}$ in [16]. However, the algorithm is modified so that the kinematics, that is strongly heterogeneous, can be captured with an acceptable noise level. 
Formulation

\section{General format}

The generic form of the optical flow equation is written as

$f(\boldsymbol{X})=g(\boldsymbol{X}+u(\boldsymbol{X}))$.

In this equation, $\boldsymbol{X}$ is the vector denoting the position of the current point with respect to the image or volume coordinates system and $u$ the unknown displacement vector field. $f$, respectively $g$, is the reference, respectively deformed, grey level function representing the image or volume of the sample. The displacement is then decomposed over finite element basis functions $\left\{\phi_{i}\right\}_{i \in[1, N]}, N$ being the number of shape functions. If the associated degrees of freedom are denoted as $u_{i}$ then the displacement is

$u(X)=\sum_{i \in[1, N]} \phi_{i} u_{i}=[\phi]\{\mathbf{U}\}$.

After this decomposition is introduced in Equation (4), a non-linear least square resolution is adopted. At each iteration, the solution increment $\mathrm{dU}$ is obtained through

$[\mathbf{M}]\{\mathrm{d} \mathbf{U}\}=\{\mathbf{b}\}$.

In this equation, $\mathrm{d} \mathbf{U}$ is a vector that collects the values of the searched increment of the set of degrees of freedom $\left\{u_{i}\right\}_{i \in[1, N]}, \mathbf{M}$ is a matrix and $\{b\}$ the driving force vector. The following expressions are adopted for the coefficients in $\mathbf{M}$ :

$M_{n m}=\iint_{\Omega}\left(\phi_{n} \cdot \nabla f\right)\left(\phi_{m} \cdot \nabla f\right) \mathrm{d} \mathbf{X}$,

and the coefficients of $\{\mathbf{b}\}$ are calculated using the following formula:

$b_{n}^{(i)}=\iint_{\Omega}\left(\phi_{n} \cdot \nabla f\right)\left(f(\mathbf{X})-g\left(\mathbf{X}+[\phi]\{\mathbf{U}\}^{(i)}\right)\right) \mathrm{d} \mathbf{X}$.

Note that the consistent tangent operator involves $\nabla g(\boldsymbol{X}+$ $u(X))$ whereas $\nabla f$ is used in Equations (7) and (8). This modification has been adopted because it allows for computing $\mathbf{M}$ once for all, only $\{b\}$ is needed to be updated at each iteration. Note that, when convergence is being reached, $\mathbf{M}$ becomes closer and closer to the consistent operator.

\section{Performances}

Based on the analysis of artificially deformed volumes, the performances of the algorithm described in the previous section are presented. The initial volume $V_{0}$ acquired during the experiment is used as a reference. This reference volume is then artificially translated by 0.5 voxel in each direction. The displacement is then evaluated using regular meshes for different element sizes (16, 32 and 64 voxels). Once the displacement has been computed, the measurement uncertainty is evaluated as the standard deviation of the displacement vector $U$ (RMS error). The measurement error is defined as the systematic error (Mean error), ie the averaged scatter between the computed displacement $U$ and the prescribed displacement $(0.5$ voxel). Figure 6 shows the evolution of these two error indicators for different element sizes. The measurement error is really low (down to $10^{-4}$ voxel) when compared to the measurement uncertainty. The uncertainty increases as the mesh size is decreased. Indeed, when the element size decreases, the number of degrees of freedom increases but not the number of voxels in the image, meaning that the ratio between the number of unknowns and

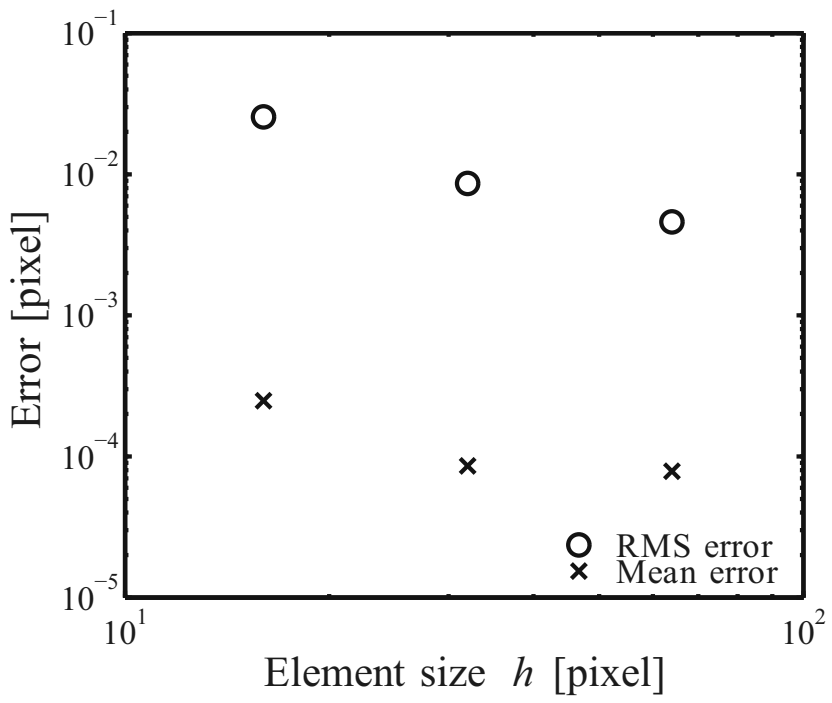

(a)

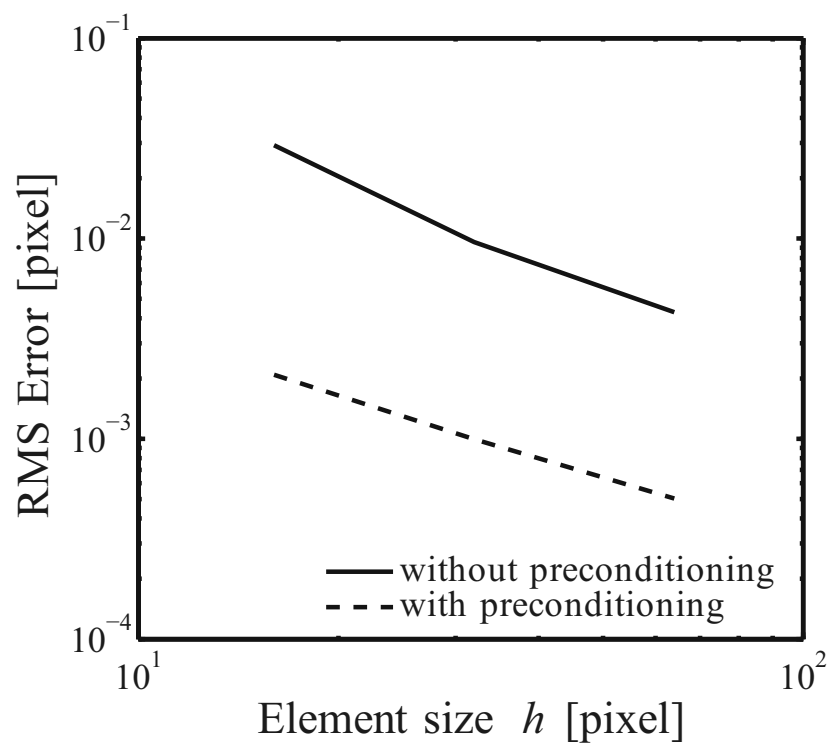

(b)

Fig. 6 (a) Mean error and RMS error obtained for an artificial rigid body translation as a function of the element size. (b) comparision of the RMS error on the $z$ component of the displacement with or without using the preconditioning 
the amount of constraint applied to the solution is decreasing. The fluctuations in $\mathbf{U}$ are thus less constrained and the uncertainty increases. Similar trends are obtained for 2D images. Note that this analysis accounts for the fluctuations induced by the algorithm only but not by the noise in the data acquisition chain. The actual uncertainty is thus expected to be higher than the value obtained herein.

In the present case, the displacement that is searched for is supposed to be discontinuous. Furthermore, the discontinuity is vanishing rapidly from the free edges of the specimen. Element sizes as small as about 6 voxels might be used to capture this strongly localized and varying displacement field. However, the levels of displacement discontinuity are not expected to exceed 2 voxels. Thus, the noise level expected with such small elements may make the analysis useless. Further, the tomographic images of the studied GFRP composite reveals an extremely anisotropic image texture. Indeed, the image gradient along the fibers is far smaller than the image gradient in orthogonal directions. In the case of perfectly orthotropic images (image gradient along one direction only), the displacement is not measurable in the directions along which the image gradient cancels out. Whereas the effect of this anisotropy has not been underlined by the analysis presented above on artificially translated images (because the texture of the real images is not perfectly orthotropic), the analysis of two real images is made more awkward by the noise introduced by the whole acquisition chain. To solve the compromise between uncertainty and resolution and the difficulties introduced by the anisotropy of the image texture, a preconditioning technique is elaborated as described in the next paragraph.

\section{Preconditioning}

From [10], the only component of the residual displacement field is along the loading direction $z$. In addition, there is no variation of the displacement field along $z$ in the unloaded state. Conversely, strong gradients are expected along $x$ at the ply interfaces and along $y$ in the vicinity of the free edges. Rigid body motions (translation and rotation) must also be considered in order to accommodate the manual positionning of the sample inside the tomograph. A displacement field corresponding to a spherical strain tensor is also added, so that the artificial affine transformation induced by the thermal dilatation of the X-ray tube can fully be accommodated [18].

To capture these effects, first, a mesh with 8-voxel elements along $x$ and varying element size along $y$ (from 64 in the center to 6 voxels at the specimen edges) is built. Note that the edges of the mesh fit the specimen edges (even if not aligned with the image frame) with a 1 to 2-voxel accuracy.

Second, the kinematic allowed by the initial mesh is constrained by using a preconditioning method. A projection operator $\mathbf{P}$ is constructed so that the motion reduces to a few degrees of freedom collected into a vector $U^{r}$. The full displacement nodal vector is then retrieved by

$\{\mathbf{U}\}=[\mathbf{P}]\left\{\mathbf{U}^{r}\right\}$.

At each iteration of the non-linear least-squares algorithm presented in the previous section, the reduced system to solve is:

$[\mathbf{P}]^{T}[\mathbf{M}][\mathbf{P}]\left\{\mathrm{d} \mathbf{U}^{r}\right\}=[\mathbf{P}]^{T}\{\mathbf{b}\}$.

In practice, $\mathbf{P}$ contains, for the present analysis, three rigid body rotations, two rigid body translations along $x$ and $y$, a spherical strain and a nodal displacement along $z$. Further, $\mathbf{P}$ cancels out the variation along $z$ of the latter field. Using this preconditioning technique the number of degrees of freedom reduces from $3 \times n_{x} \times n_{y} \times n_{z}\left(n_{x}, n_{y}, n_{z}\right.$ being the number of nodes along the $x, y$ and $z$ direction respectively) to $3+2+1+n_{x} \times n_{y}$. At the macroscopic scale $((y, z)$ plane $)$, the pixel size is about ten times higher than the pixel size at the mesoscopic scale $((x, z)$ plane) while the amplitude of the displacement in physical units is the same.Consequently, the proposed preconditioning technique is also used for the DIC analysis of the residual state at the macroscopic scale. The uncertainty analysis is also performed using the preconditioning technique proposed above. The RMS error is computed on the $z$ component only. Indeed, as the rigid translations along $x$ or $y$ are captured with only one degree of freedom their uncertainty vanishes. In Fig. 6(b), the RMS errors with or without preconditioning are plotted as functions of the element size. The preconditioning allows for decreasing the uncertainty by a factor of about 10 . It might thus be possible to solve the uncertainty/resolution compromise by introducing a priori knowledge about the searched displacement field in the spirit of so-called Integrated approaches that use closed-form solutions eg, for cracks [20]. A mesh sufficiently refined in the vicinity of the ply interfaces and around the free edges can be used in order to track the strong gradient of the displacement field while the projection operation keeps the number of degrees of freedom (and thus the noise level) as small as possible.

\section{Results and Discussion}

In this section, 2D and 3D displacement fields are examined. A good agreement between both full field measurements is obtained and the in depth information provided by the $3 \mathrm{D}$ analysis is detailed.

Figure 7 shows the displacement fields in the loading direction obtained by DIC from the mesoscopic and macroscopic scales. For the mesoscopic scale, Fig. 7(a), (c), (e) and $(\mathrm{g})$, a regular mesh of $32 \times 32$ pixels quadrangles is 
Fig. 7 Displacement fields, over different planes, along the loading axis $(z)$ in $\mu \mathrm{m}$ under loading (5000 N and $6000 \mathrm{~N}$ ) and after unloading

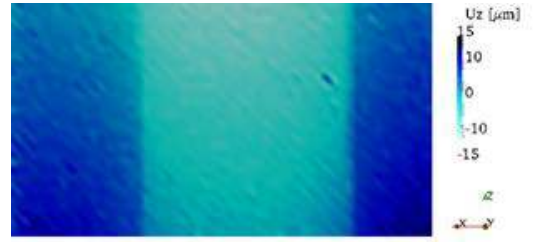

(a) $(x, z), 5000 \mathrm{~N}$, loading

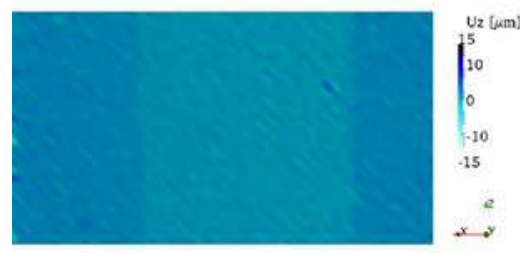

(c) $(x, z), 5000 \mathrm{~N}$, unloading

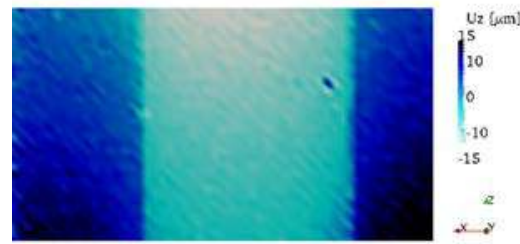

(e) $(x, z), 6000 \mathrm{~N}$, loading

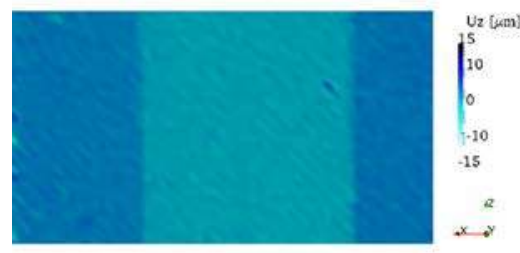

(g) $(x, z), 6000 \mathrm{~N}$, unloading

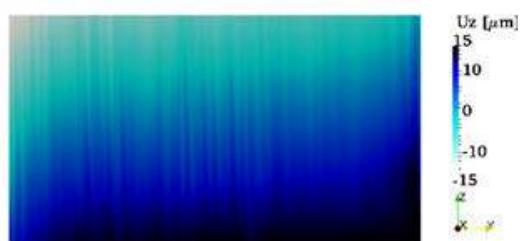

(b) $(y, z), 5000 \mathrm{~N}$, loading

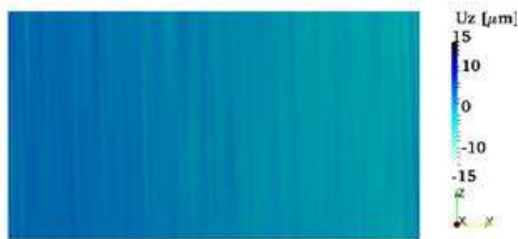

(d) $(y, z), 5000 \mathrm{~N}$, unloading

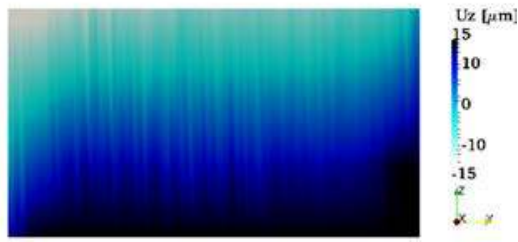

(f) $(y, z), 6000 \mathrm{~N}$, loading

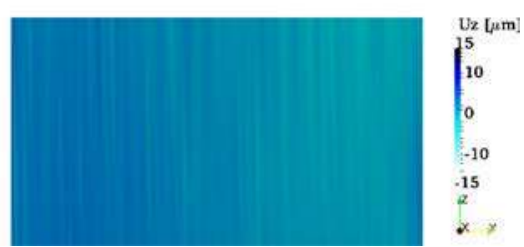

(h) $(y, z), 6000 \mathrm{~N}$, unloading used. The images are analyzed within a zone that covers the sample thickness in the $x$ direction and $1.5 \mathrm{~mm}$ in the $z$ direction. This corresponds to a zone of about $1800 \times 1000$ pixels. For the macroscopic scale, the results of Fig. 7(b), (d), (f) and (h) have been obtained using a regular mesh of quadrangles which edges are 8 pixels in the $y$ direction and 256 pixels in the $z$ direction, ie about $720 \times 1400$ pixels. The analysis is performed over $15.5 \mathrm{~mm}$ in the $z$ direction whereas the entire sample width is analyzed. Note that for illustration purposes, the mesh has been shrinked to have the same aspect ratio as for the mesoscopic analysis in the corresponding Figures.

In Fig. 7(a), (b), (e) and (f), the displacements measured under loading at $5000 \mathrm{~N}$ and $6000 \mathrm{~N}$ are depicted. These displacement fields result from the superposition of a constant axial strain and of localized strains at the interfaces between plies of different orientations for the mescoscopic fields and of a localized strain in the vicinity of the sample boundaries for the macroscopic fields. For the mesoscopic fields, this results in sharp variations of the displacement component along the loading axis at the interfaces between plies of different orientations. For the macroscopic field, sharp variations are obtained close to the sample free surface. These results fully agree with the results in the literature [7]. No significant difference on the shape of the displacement field is obtained by increasing the load from $5000 \mathrm{~N}$ to $6000 \mathrm{~N}$. In Fig. 7(c), (d), (g), and $(\mathrm{h})$, the residual displacement fields are plotted (ie 
the displacement when the loading is decreased to $0 \mathrm{~N}$ ). In Fig. 7(c), the displacement map shows a very low displacement magnitude (about $0.5 \mu \mathrm{m}$ difference between the central plies and the external plies) meaning that the specimen has almost recovered its initial configuration. However, in Fig. 7(g), it is clearly observed that a residual displacement field is appearing even if no non-linearity is observed on the macroscopic stress-strain curve (see Fig. 2). This residual displacement field is discontinuous at the interfaces between plies of different orientations. A displacement discontinuity of about $3.5 \mu \mathrm{m}$ persists after unloading from $6000 \mathrm{~N}$. This is interpreted as a consequence of the initiation of delamination as proposed in [9]. Considering the difference in the physical pixel size between the images at the mesoscopic scale and those at the macroscopic scale that is ten times higher, analyzing the latter at the residual state becomes awkward. In the following, the preconditioning technique is used for analysing the residual displacements at the macroscopic scale. The $2 \mathrm{D}$ mesh that is used for these analyses is extracted from the surface of the 3D mesh used for DVC and then adapted, using the appropriate scaling factor, to the pixel size of the 2D macroscopic images. The element size variation along $y$ is thus the same as for the $3 \mathrm{D}$ analysis. The allowed kinematics consists in a rigid translation along $y$, a rigid rotation along $x$, and a displacement along $z$ varying along $y$.

The residual displacement states are now characterized in $3 \mathrm{D}$ by using the methodology proposed in the previous section. Two residual states are analyzed: after a loading at $6000 \mathrm{~N}$ and after a loading at $7000 \mathrm{~N}$. At $8000 \mathrm{~N}$, failure of the sample occurs and at $5000 \mathrm{~N}$ the magnitude of the residual displacements that may be tracked for is too low. Indeed, a displacement discontinuity as low as $0.5 \mu \mathrm{m}$ (see Fig. 7(c)) is observed at $5000 \mathrm{~N}$ which is below the resolution of the tomographic images $(4.5 \mu \mathrm{m}$ being the physical size of a voxel). For these reasons only the residual states after $6000 \mathrm{~N}$ and $7000 \mathrm{~N}$ loads are analyzed in 3D. Note that for the two analyzed states, the dilatation compensation degree of freedom included in the reduced basis were -0.0001 and 0.0003 respectively. This means that the warmup phase before each scan was able to cancel out the effect of X-ray source temperature variation during and between scans. Figure 8 shows the comparison of the displacement in the loading direction obtained from a 3D analysis with the ones obtained from the 2D analyses after a $6000 \mathrm{~N}$ load. The 2D measurements are performed on the free surface of the sample in the $(x, z)$ and $(y, z)$ planes. They are thus compared to the $3 \mathrm{D}$ fields on the two corresponding mesh
Fig. 8 Comparison, for different planes, of the residual displacement (in $\mu \mathrm{m}$ ) along the loading axis after unloading from $6000 \mathrm{~N}$. The field on the left is obtained by DVC on the $3 \mathrm{D}$ images and the field on the right is obtained by 2 D DIC
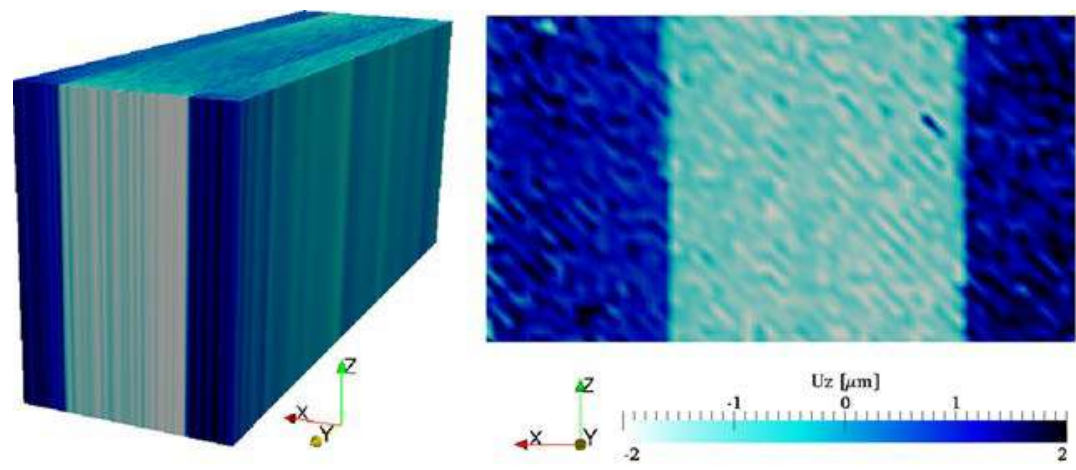

(a) $(x, z)$, DVC v.s. DIC
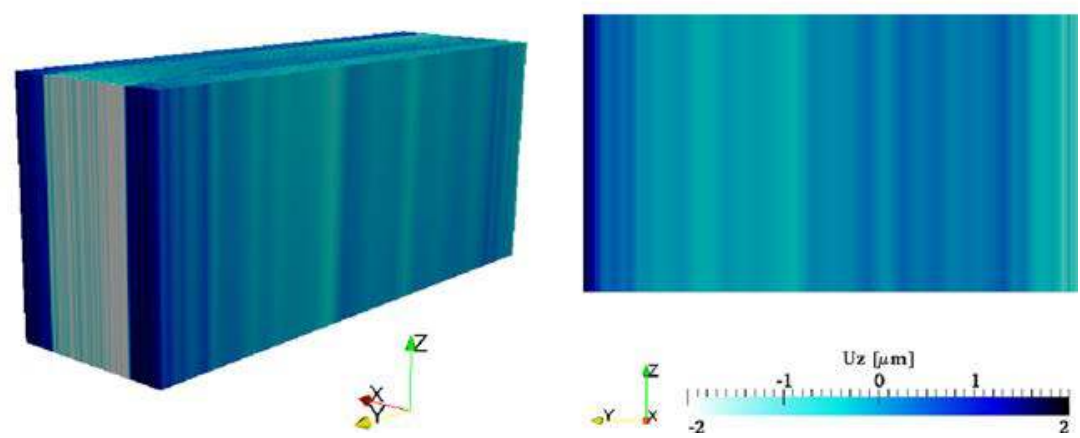

(b) $(y, z)$, DVC v.s. DIC 
surfaces. In Fig. 8(a), a discontinuity at the interfaces between plies of different orientations is obtained in the 3D residual displacement as in 2D. The displacement magnitudes are in good agreement. It is also observed, from the 3D measurements, that strong variations of the displacement field are obtained in the witdh (along $y$ ) of the specimen in the vicinity of the edges. Figure 8 shows the comparison of these variations in the $(y, z)$ plane observed at mesoscopic scale and the corresponding surface extracted from 3D measurements. Again, a good agreement is obtained.

At mesoscopic scale, an average of the 2D displacement along the loading direction $z$ is performed in order to allow an easier interpretation of the experimental measurements and comparison between $2 \mathrm{D}$ and $3 \mathrm{D}$ results. The $3 \mathrm{D}$ fields do not need to be averaged as the preconditioning has a priori canceled the variations of the displacement field along $z .2 \mathrm{D}$ and $3 \mathrm{D}$ residual displacements, on the specimen edge $(x, z)$, averaged along $z$ are plotted in Fig. 9 as a function of $x$. For the two residual states (after $6000 \mathrm{~N}$ and $7000 \mathrm{~N}$ ), the results from the $2 \mathrm{D}$ and the $3 \mathrm{D}$ analysis are consistent. The residual displacement is nearly constant in each ply and a displacement discontinuity is obtained at the interfaces between plies of different orientations. The level of this displacement jump is about $3.5 \mu \mathrm{m}$ after $6000 \mathrm{~N}$ and $6.5 \mu \mathrm{m}$ after $7000 \mathrm{~N}$.

The 3D analyses allow measuring the evolution of the displacement discontinuity along the $y$ direction, in depth, from the free edge to the center of the specimen. Figure 10 shows the evolution of the residual displacement after the $6000 \mathrm{~N}$ loading. As expected, the discontinuous displacement field observed on the free surface of the specimen rapidly vanishes in depth. For evaluating the localized nature of the residual displacement, Fig. 11(a) shows the evolution of the displacement discontinuity (evaluated as

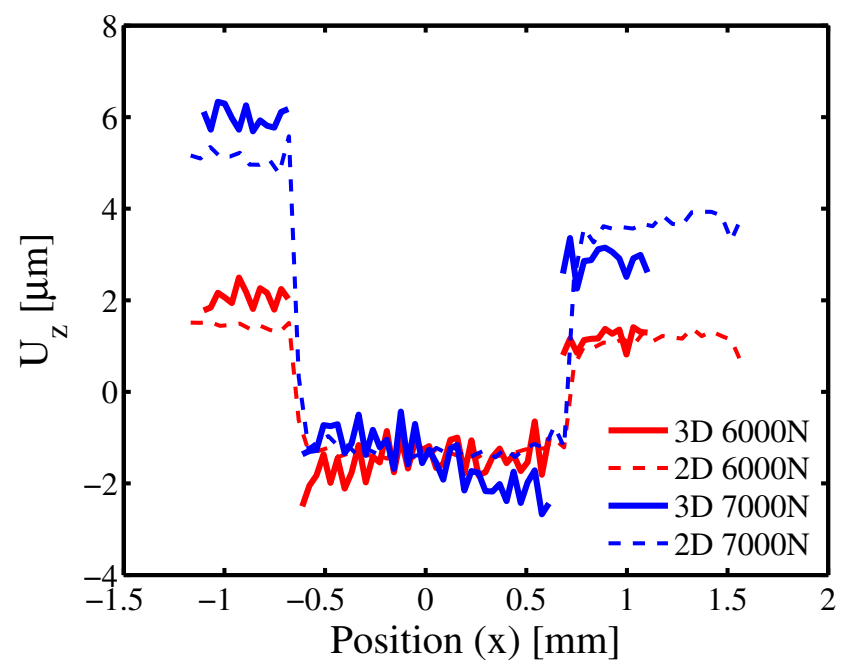

Fig. 9 Comparison of the averaged residual displacement along the loading direction in $\mu \mathrm{m}$

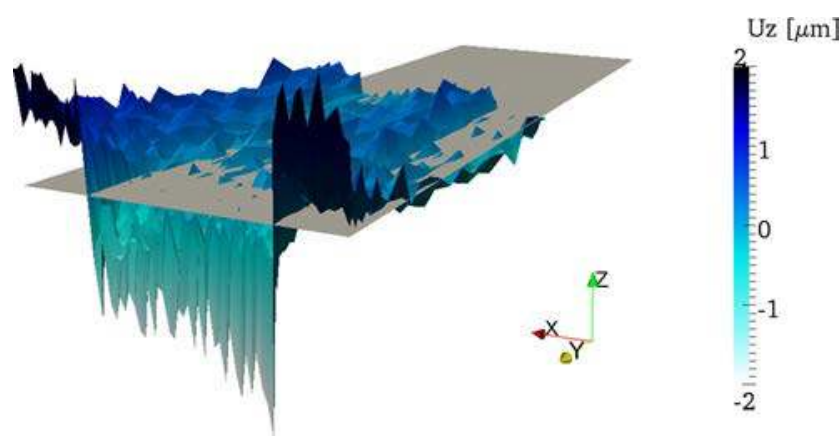

Fig. 10 Evolution, within the half of the studied volume, of the residual displacement along the loading axis

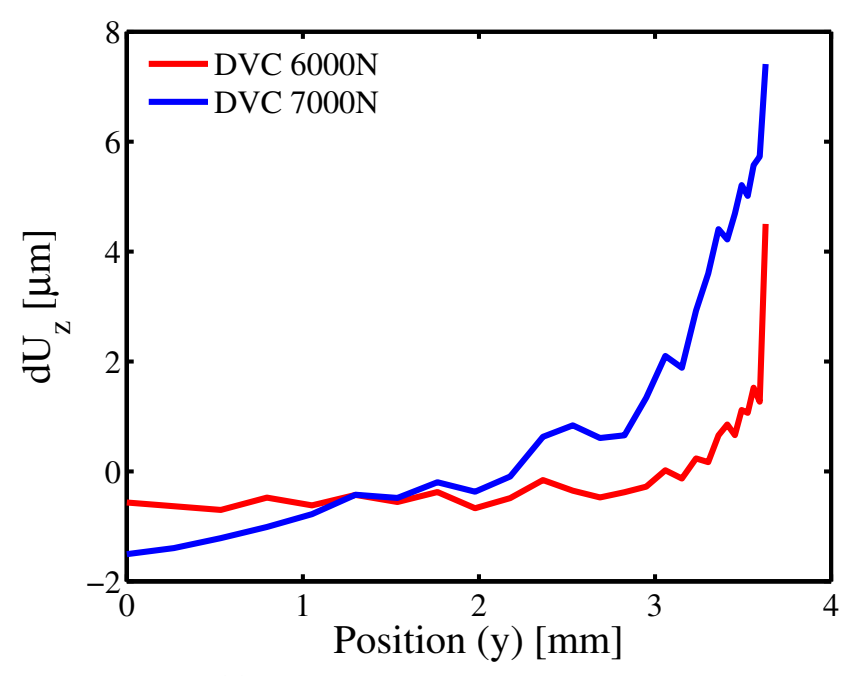

(a) DVC $d U_{z}$ along a $+15^{\circ} /-15^{\circ}$ interface

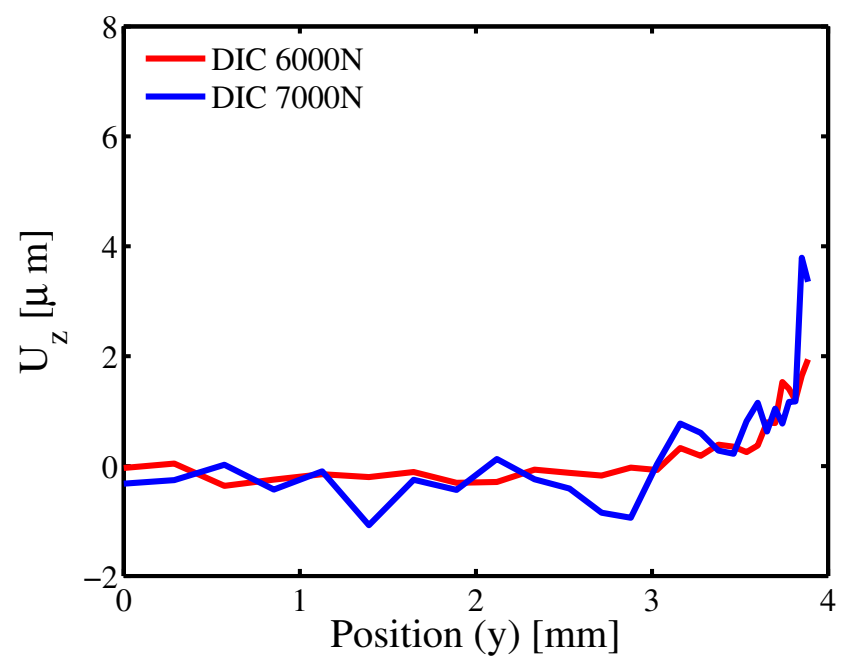

(b) DIC averaged $U_{z}$ on a free $(y, z)$ plane

Fig. 11 Evolution of: (a) the displacement jump along a $+15^{\circ} /-15^{\circ}$ interface obtained from DVC and (b) the averaged displacement on a free $(y, z)$ plane obtained by DIC 
the difference of the displacement between two neighboor lines of the mesh) at the ply interface as a function of the distance to the center of the sample. Far from the center, ie at the free-edge, the above-mentioned displacement discontinuity (measured on the free $(x, z)$ surface in 2D or $3 \mathrm{D})$ is recovered. The displacement discontinuity is localized in the vicinity of the specimen surface. Close to the center, displacement jumps, which are considered in the measurement uncertainty, are obtained. Increasing the load induces an amplification of the magnitude of the residual displacement discontinuity at the interlaminar interface and a propagation of delamination in depth. The in-depth signature of the delamination can roughly be estimated to the ply thickness and 1.5 times the ply thickness for the $6000 \mathrm{~N}$ and $7000 \mathrm{~N}$ loads respectively. The residual displacement fields that we are able to capture are due to the delamination of the ply interfaces plus the accumulation of the irreversible strain along and at the tip of the delaminated interfaces. The displacement discontinuity depicted in Fig. 11(a) can also be compared to the residual displacement measured at macroscopic scale on the sample surface, in the $(y, z)$ plane. Figure 11(b) shows the variation of the 2D macroscopic displacement obtained with preconditioning hypothesis as a function of the distance $y$ from the specimen edge. Note that for the images at the macroscopic scale, the pixel size is $11 \mu \mathrm{m}$, the displacement level plotted in Fig. 11(b) are thus of about $1 / 5$ of pixel. However, the same trends as in Fig. 11(a) are obtained: the residual displacement is localized in the vicinity of the specimen edges, the magnitude of the load changes the magnitude of the residual field and, whereas less obvious than for the displacement jump (due to the very low displacement levels to be measured), the region affected by a residual displacement enlarges with the load. Considering the symmetry of the stacking sequence and as illustrated by Fig. 10, the residual displacement in the two plies in the specimen center is the opposite of the displacement in the external plies. As the residual displacement has been shown to be almost constant along the thickness $x$ in each ply, this means that the displacement jump is expected to be twice the displacement within the plies and thus twice the displacement on the specimen $(y, z)$ surface. This is confirmed by comparing Fig. 11(a) and (b).

In the literature, several publications show that the indepth influence of free-edge effects has an exponential shape and that a stress singularity is obtained. However, the consequence of this extremely confined effect has not already been studied in 3D to our knowledge. In the present case, delamination occurs in mode III and thus no opening of the ply interface could be observed. Figure 12 shows a micrography of the specimen surface after the $7000 \mathrm{~N}$ load. The fracture interface is observed at the interlaminar interface. The thickness of the fracture band is roughly estimated to be about $0.5 \mu \mathrm{m}$ which is far below the resolution of the

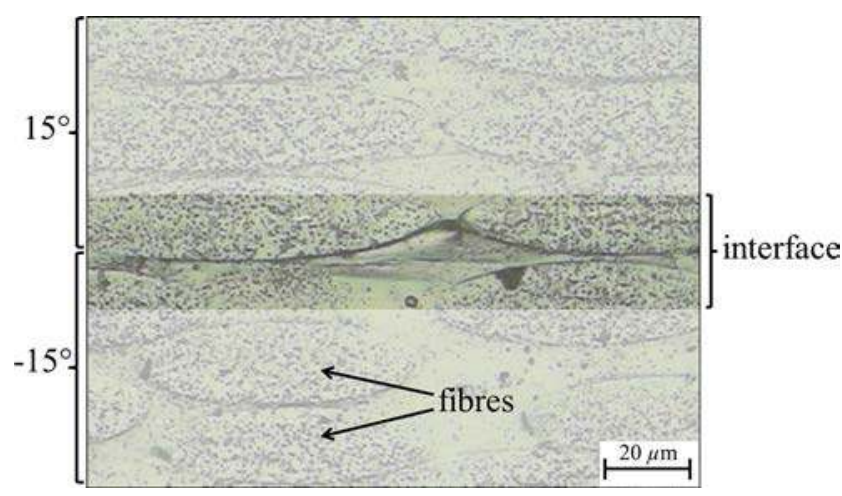

Fig. 12 Micrography of an interlaminar interface after the $7000 \mathrm{~N}$ load

$\mathrm{X}$-ray tomography images. Further as the fracture occurs in mode III (no opening, only sliding), there is no guaranty that delamination could have been observed with a higher resolution of the 3D images. However, using X-ray tomography combined with a suitable DVC algorithm, adapted to highly localized phenomena, it has been possible to observe the 3D propagation of delamination at interlaminar interfaces, from the free-edge to the interior of the sample, by characterizing the residual displacement states.

\section{Conclusion}

Edge effects in laminated structures induce strong stress and strain concentrations at interlaminar interfaces between plies having different orientations due to the material properties discontinuity. This leads to complex tridimensional mechanical states which require tridimensional characterization techniques. An investigation of edge effects by coupling 2D and 3D measurements has been performed on Glass Fibre Reinforced Polymer composite with angle ply stacking sequence $\left[15^{\circ} /-15^{\circ}\right]_{s}$.

An experimental protocol allowing to perform in situ 2D measurements as well as 3D X-Ray characterization with residual displacement measurements has been defined. A Digital Volume Correlation algorithm has been proposed with a priori preconditioning hypothesis to assess these highly localized displacement gradients on such an anisotropic texture. This DVC strategy has been validated by comparison with the $2 \mathrm{D}$ measured displacement fields on the specimen edges.

After unloading, 2D and 3D residual displacement fields show a nearly constant displacement in each ply and a displacement discontinuity at interlaminar interface which results from delamination initiation and accumulation of irreversible strains near the delaminated interlaminar interface. This interpretation has been validated by observations of delamination micro-cracks on edges of the samples. The 
in depth variation of this displacement jump has been highlighted at unloading step. This variation is observed to have an exponential nature with displacement jump at sample edges vanishing at the sample centre. The increase of the magnitude of displacement jump with the applied load has been highlighted as well as its in depth propagation. Thus, this experimental protocol as well as the defined DVC strategy have allowed to evidence the initiation and the propagation of the delamination in depth. The results seem to highlight that the 2D macroscopic displacement gradients measured near edges at residual states are consistent with 3D measurements and might give a good estimation of the in depth influence of the delamination. In such protocol with monotonic loading, the propagation of the delamination becomes unstable when the critical load is reached. It would be interesting in following works to extend this study to cyclic loadings in order to, on the one hand, have a more stable delamination propagation and, on the other hand, apply loadings more representative of the ones occuring in real life conditions. The experimental protocol coupling in situ 2D measurements and 3D observations and measurements should allow to highlight and follow delamination propagation under cyclic loadings.

Acknowledgements The lab composite of the ENSAM Lille is gratefully acknowledged for the preparation of the samples. The MATEIS laboratory at INSA Lyon is also gratefully acknowledged for the use of the X-ray laboratory tomography device.

\section{References}

1. Herakovich C (1981) On the relationship between engineering properties and delamination of composite materials. J Compos Mater 15:336-348

2. Pagano N, Pipes R (1971) The influence of stacking sequence on laminate strength. J Compos Mater 5(1):50-57

3. Kant T, Swaminathan K (2000) Estimation of transverse/ interlaminar stresses in laminated composites - a selective review and survey of current developments. Compos Struct 49(1): 65-75

4. Mittelstedt C, Becker W (2004) Interlaminar stress concentrations in layered structures: Part i-a selective literature survey on the freeedge effect since 1967. J Compos Mater 38(12):1037-1062
5. Nosier A, Maleki M (2008) Free-edge stresses in general composite laminates. Int J Mech Sci 50(10):1435-1447

6. Czarnek R, Post D, Herakovich C (1983) Edge effects in composites by moire interferometry. Exp Tech 7(1):18-21

7. Herakovich CT, Post D, Buczek M, Czarnek R (1985) Free edge strain concentrations in real composite laminates: experimentaltheoretical correlation. J Appl Mech 52(4):787-793

8. Mollenhauer D, Reifsnider K (2000) Measurements of interlaminar deformation along the cylindrical surface of a hole in laminated composite materials by moiré interferometry. Compos Sci Technol 60(12):2375-2388

9. Lecomte-Grosbras P, Paluch B, Brieu M, De Saxcé G, Sabatier L (2009) Interlaminar shear strain measurement on angle-ply laminate free edge using digital image correlation. Compos Part A: Appl Sci Manuf 40(12):1911-1920

10. Lecomte-Grosbras P, Paluch B, Brieu M (2013) Characterization of free edge effects: influence of mechanical properties, microstructure and structure effects. J Compos Mater 47(22):2823-2834

11. Kim R, Soni S (1984) Experimental and analytical studies on the onset of delamination in laminated composites. J Compos Mater 18(1):70-80

12. Lorriot T, Marion G, Harry R, Wargnier H (2003) Onset of freeedge delamination in composite laminates under tensile loading. Compos Part B: Eng 34(5):459-471

13. Gilchrist M, Svensson N (1995) A fractographic analysis of delamination within multidirectional carbon/epoxy laminates. Compos Sci Technol 55(2):195-207

14. Takeda N, Kobayashi S, Ogihara S, Kobayashi A (1999) Effects of toughened interlaminar layers on fatigue damage progress in quasi-isotropic cfrp laminates. Int J Fatigue 21(3):235-242

15. Schilling PJ, Karedla BR, Tatiparthi AK, Verges MA, Herrington PD (2005) X-ray computed microtomography of internal damage in fiber reinforced polymer matrix composites. Compos Sci Technol 65(14):2071-2078

16. Roux S, Hild F, Viot P, Bernard D (2008) Three-dimensional image correlation from x-ray computed tomography of solid foam. Compos Part A: Appl Sci Manuf 39(8):1253-1265

17. Salvo L, Suéry M, Marmottant A, Limodin N, Bernard D (2010) $3 \mathrm{~d}$ imaging in material science: Application of $\mathrm{x}$-ray tomography. Comptes Rendus Physique 11(9):641-649

18. Limodin N, Réthoré J, Adrien J, Buffière JY, Hild F, Roux S (2011) Analysis and artifact correction for volume correlation measurements using tomographic images from a laboratory $\mathrm{x}$-ray source. Exp Mech 51(6):959-970

19. Besnard G, Hild F, Roux S (2006) 'finite-element' displacement fields analysis from digital images: Application to portevin-le châtelier bands. Exp Mech 46(6):789-803

20. Roux S, Hild F (2006) Stress intensity factor measurement from digital image correlation: post-processing and integrated approaches. Int J Fract 140(1-4):141-157 